SCIENCE CHINA

Physics, Mechanics \& Astronomy

\title{
Atomistic insights into plasmon induced water splitting
}

\author{
Lei Yan, and Sheng Meng* \\ Institute of Physics, Chinese Academy of Sciences, Beijing 100190, China
}

Received November 7, 2016; accepted November 10, 2016; published online November 28, 2016

\begin{abstract}
Citation: $\quad$ L. Yan, and S. Meng, Atomistic insights into plasmon induced water splitting, Sci. China-Phys. Mech. Astron. 60, 027032 (2017), doi: 10.1007/s11433016-0442-6
\end{abstract}

Photosynthesis supports the existence of life on earth. At its heart is a remarkable chemical reaction at the ambient condition: water splitting. Producing hydrogen by artificial photosynthesis has been considered as one way to potentially solve the world's ever-increasing energy demands [1]. In 1972, Fujishima and Honda [2] firstly realized photochemical splitting of water on $\mathrm{TiO}_{2}$ electrodes using UV light. In $\mathrm{TiO}_{2}$, an absorbed photon creates an electron-hole pair. The electrons drive reduction of hydrogen ions to produce $\mathrm{H}_{2}$ gas, while the holes drive oxidation of hydroxyl ions to produce $\mathrm{O}_{2}$ gas. However, $\mathrm{TiO}_{2}$ with an optical band gap of $\sim 3.2 \mathrm{eV}$ does not absorb light in the visible region, which is most abundant in solar spectrum. Several attempts to extend the cutoff wavelength by doping and searching for new materials with a smaller bandgap would improve the efficiency of energy harvest in the visible range [3].

Plasmon-enhanced solar water splitting has gained increasing attention thanks to its potential to drastically improve energy conversion efficiency [4]. The unique property of noble metal nanoparticles (NP) to absorb and scatter light at specific wavelengths across a wide range of optical spectrum, makes them excellent catalyst for photochemistry. The use of plasmon-enhanced water splitting falls primarily into two categories: indirect and direct photocatalysis. Indirect photocatalysis is prevalent in literature, which combines an oxide semiconductor with plasmonic metal nanostructures as cocatalyst [5]. In this scenario, localized surface plasmon of metal NPs is initially optically excited at a specific frequency, then

*Corresponding author (email: smeng@iphy.ac.cn) decays into hot electrons. Only hot electrons with sufficient energy to overcome the Schottky barrier can be collected by the conduction band of the semiconductor, and be used to drive the photocatalytic reaction. The charge transfer bottleneck significantly limits reaction efficiency.

To conquer this bottleneck, another category called direct photocatalysis, where plasmonic NPs act as both light absorbers and catalytic active sites, is proposed. This strategy allows for low-intensity visible photons to drive catalytic reactions on plasmonic NP surfaces [6]. Robatjazi et al. [7] demonstrated solar water splitting in a Schottky-free junction driven by direct injection of hot electrons from gold NP to molecules. So far, these experiments show a rather low photocatalytic activity improper for practical use. The key to achieve a significant efficiency improvement is strong light absorption and efficient carrier separation. More importantly, the microscopic mechanism for photocatalytic water splitting, especially its dynamic processes at the atomic scale, has been elusive, inhibiting further efficiency improvement in experiment.

Yan et al. [8] stride to attack this problem. They investigated real time dynamics of water splitting on Au NPs (diameter $D=1.6-2.1 \mathrm{~nm}$ ) irradiated by femtosecond laser pulses, by using time-dependent density functional theory. Ultrafast time evolution of the occupied orbitals of water molecule on gold NP $(D=1.9 \mathrm{~nm})$ under laser illumination were demonstrated, as shown in Figure 1. Under the laser pulse, the water molecule first rotates itself to align with field direction and then one of $\mathrm{H}$ atoms is detached from the $\mathrm{O}$ atom. Laser-induced hot electron generation and accumulation in the anti- 


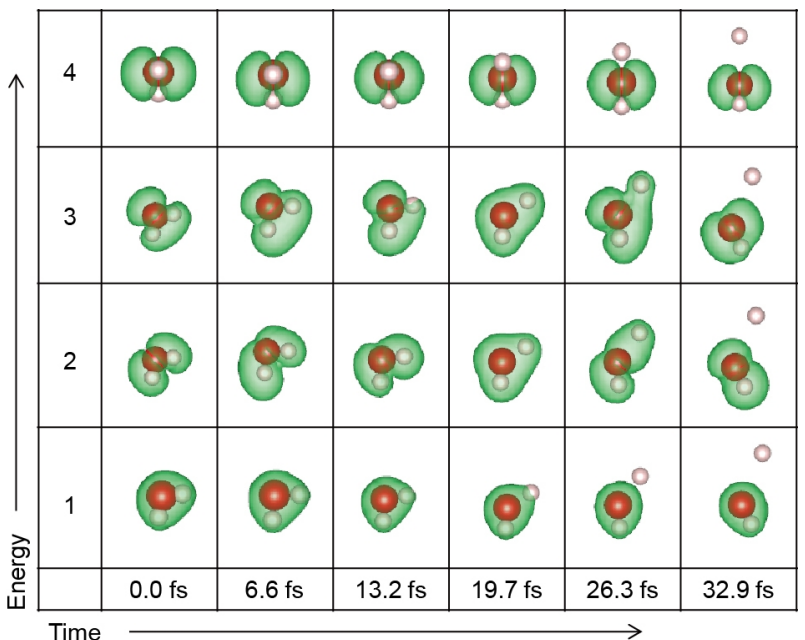

Figure 1 (Color online) Snapshots of time evolution of occupied orbital density for water molecule on gold NP exposed to pulsed laser at the frequency of $2.62 \mathrm{eV}$ with a pulse width of $10 \mathrm{fs}$ and amplitude of $1.6 \mathrm{~V} / \AA$.

bonding state of water were directly observed in first-principles dynamic simulations. These observations provide compelling evidence that occupation of anti-bonding orbital in water upon laser excitation leads to water splitting.

To demonstrate the reaction is indeed plasmon-induced, Yan et al. [8] also showed wavelength dependent reaction rates for different Au NP sizes. For the diameter of $D=1.6$ $\mathrm{nm}$, the rate compares well with the trend in absorption spectrum, in good agreement with experimental measurements. However, in the case of $D=1.9 \mathrm{~nm}$, reaction rate for excitation at low-absorption peak is actually higher than that for high-absorption peak - a surprising discovery indicative of mode selectivity. Similar behavior occurs for NPs with larger diameters $(D=2.1 \mathrm{~nm})$. Therefore, the rate of water splitting is dependent not only on respective optical absorbance, but also on the mode of plasmon excitation. The low-absorption peak has odd oscillation periods across the NP region (odd mode), and the high-absorption peak has even oscillation periods (even mode). Upon photoexcitation, hot electrons in the odd mode have better energy overlap with the anti-bonding state of water. Thus, aligning the energy level of hot electrons with the anti-bonding orbital level of adsorbed molecule would ultimately determine reaction rates of photocatalytic processes.

The rate of plasmon-induced water splitting can be tuned by varying the NP size, shape, material, and proximity to other NPs [9]. Future studies can take these advantages for further improvements. For instance, by combining nanorods of various aspect ratios to create panchromatic absorbers covering most of the useful solar spectrum, the estimated quantum efficiency can increase to $\sim 0.1 \%$ [10]. $\mathrm{MoS}_{2}$ monolayer with deposited Au@Ag nanorattles also effectively enhanced the hydrogen evolution reaction efficiency [11]. Most photocatalysts are rare and expensive, and the search for cheap newtype photocatalysts is essential $[12,13]$. Liu et al. [14] report the fabrication of a metal-free carbon nanodot-carbon nitride $\left(\mathrm{C}_{3} \mathrm{~N}_{4}\right)$ nanocomposite for solar water splitting, with an overall solar energy conversion efficiency of $2.0 \%$. Sprick et al. [15] demonstrate a serial of organic porous polymers which can catalyze hydrogen production from water and can be easily tuned.

In summary, two categories of plasmon-enhanced water splitting are widely investigated. Mode selectivity in the rate of water splitting results from better energy overlap between hot electrons and anti-bonding orbital of water molecule. Further studies are needed in order to fully understand the underlying mechanism and to optimize this reaction for maximizing solar energy conversion efficiency.

This work was supported by the National Key Research and Development Program of China (Grant Nos. 2016YFA0300902, and 2015CB921001), the National Natural Science Foundation of China (Grant No. 11574365).

1 J. Luo, J. H. Im, M. T. Mayer, M. Schreier, M. K. Nazeeruddin, N. G. Park, S. D. Tilley, H. J. Fan, and M. Gratzel, Science 345, 1593 (2014).

2 A. Fujishima, and K. Honda, Nature 238, 37 (1972).

3 W. Hou, and S. B. Cronin, Adv. Funct. Mater. 23, 1612 (2013).

4 M. L. Brongersma, N. J. Halas, and P. Nordlander, Nat. Nanotech. 10, 25 (2015).

5 S. Mubeen, J. Lee, N. Singh, S. Krämer, G. D. Stucky, and M. Moskovits, Nat. Nanotech. 8, 247 (2013).

6 M. J. Kale, T. Avanesian, and P. Christopher, ACS Catal. 4, 116 (2014).

7 H. Robatjazi, S. M. Bahauddin, C. Doiron, and I. Thomann, Nano Lett. 15, 6155 (2015).

8 L. Yan, F. Wang, and S. Meng, ACS Nano 10, 5452 (2016).

9 X. R. Tian, L. M. Tong, and H. X. Xu, Sci. China-Phys. Mech. Astron. 56, 2327 (2013).

10 S. Mubeen, J. Lee, D. Liu, G. D. Stucky, and M. Moskovits, Nano Lett. 15, 2132 (2015).

11 Y. Kang, Y. Gong, Z. Hu, Z. Li, Z. Qiu, X. Zhu, P. M. Ajayan, and Z. Fang, Nanoscale 7, 4482 (2015).

12 J. Zhang, J. Ren, H. X. Fu, Z. J. Ding, H. Li, and S. Meng, Sci. ChinaPhys. Mech. Astron. 58, 106801 (2015).

13 Y. P. Jia, L. W. Guo, W. Lu, Y. Guo, J. J. Lin, K. X. Zhu, L. L. Chen, Q. S. Huang, J. Huang, Z. L. Li, and X. L. Chen, Sci. China-Phys. Mech. Astron. 56, 2386 (2013).

14 J. Liu, Y. Liu, N. Liu, Y. Han, X. Zhang, H. Huang, Y. Lifshitz, S. T. Lee, J. Zhong, and Z. Kang, Science 347, 970 (2015).

15 R. S. Sprick, J. X. Jiang, B. Bonillo, S. Ren, T. Ratvijitvech, P. Guiglion, M. A. Zwijnenburg, D. J. Adams, and A. I. Cooper, J. Am. Chem. Soc. 137, 3265 (2015). 